Swarthmore College

Works

$12-3-2015$

\title{
On The Stellar Companion To The Exoplanet Hosting Star 30 Arietis B
}

S. R. Kane

T. Barclay

M. Hartmann

A. P. Hatzes

Eric L. N. Jensen

Swarthmore College, ejensen1@swarthmore.edu

See next page for additional authors

Follow this and additional works at: https://works.swarthmore.edu/fac-physics

Part of the Astrophysics and Astronomy Commons

Let us know how access to these works benefits you

\section{Recommended Citation}

S. R. Kane, T. Barclay, M. Hartmann, A. P. Hatzes, Eric L. N. Jensen, D. R. Ciardi, D. Huber, J. T. Wright, and E. V. Quintana. (2015). "On The Stellar Companion To The Exoplanet Hosting Star 30 Arietis B".

Astrophysical Journal. Volume 815, Issue 1. DOI: 10.1088/0004-637X/815/1/32

https://works.swarthmore.edu/fac-physics/245

This work is brought to you for free by Swarthmore College Libraries' Works. It has been accepted for inclusion in Physics \& Astronomy Faculty Works by an authorized administrator of Works. For more information, please contact myworks@swarthmore.edu. 
Authors

S. R. Kane, T. Barclay, M. Hartmann, A. P. Hatzes, Eric L. N. Jensen, D. R. Ciardi, D. Huber, J. T. Wright, and E. V. Quintana 


\title{
ON THE STELLAR COMPANION TO THE EXOPLANET HOSTING STAR 30 ARIETIS B
}

\author{
Stephen R. Kane ${ }^{1}$, Thomas Barclay ${ }^{2,3}$, Michael Hartmann ${ }^{4}$, Artie P. Hatzes ${ }^{4}$, Eric L. N. Jensen ${ }^{5}$, David R. Ciardi $^{6}$, \\ DANiEl Huber ${ }^{7,8}$, Jason T. Wright ${ }^{9,10}$, AND Elisa V. Quintana ${ }^{2}$ \\ ${ }^{1}$ Department of Physics \& Astronomy, San Francisco State University, 1600 Holloway Avenue, San Francisco, CA 94132, USA; skane@ sfsu.edu \\ ${ }^{2}$ NASA Ames Research Center, M/S 244-30, Moffett Field, CA 94035, USA \\ ${ }^{3}$ Bay Area Environmental Research Institute, 596 1st Street West, Sonoma, CA 95476, USA \\ ${ }^{4}$ Thüringer Landessternwarte, D-07778 Tautenburg, Germany \\ ${ }^{5}$ Department of Physics \& Astronomy, Swarthmore College, Swarthmore, PA 19081, USA \\ ${ }^{6}$ NASA Exoplanet Science Institute, Caltech, MS 100-22, 770 South Wilson Avenue, Pasadena, CA 91125, USA \\ ${ }^{7}$ Sydney Institute for Astronomy (SIfA), School of Physics, University of Sydney, NSW 2006, Australia \\ ${ }^{8}$ SETI Institute, 189 Bernardo Avenue, Mountain View, CA 94043, USA \\ ${ }^{9}$ Department of Astronomy and Astrophysics, Pennsylvania State University, 525 Davey Laboratory, University Park, PA 16802, USA \\ ${ }^{10}$ Center for Exoplanets \& Habitable Worlds, Pennsylvania State University, 525 Davey Laboratory, University Park, PA 16802, USA \\ Received 2014 December 16; accepted 2015 November 4; published 2015 December 3
}

\begin{abstract}
A crucial aspect of understanding planet formation is determining the binarity of the host stars. Results from radial velocity (RV) surveys and the follow-up of Kepler exoplanet candidates have demonstrated that stellar binarity certainly does not exclude the presence of planets in stable orbits and the configuration may in fact be relatively common. Here we present new results for the 30 Arietis system which confirms that the B component hosts both planetary and stellar companions. Keck AO imaging provides direct detection of the stellar companion and additional RV data are consistent with an orbiting star. We present a revised orbit of the known planet along with photometry during predicted transit times. Finally, we provide constraints on the properties of the stellar companion based on orbital stability considerations.
\end{abstract}

Key words: planetary systems - stars: individual (30 Ari B) - techniques: high angular resolution - techniques: photometric - techniques: radial velocities

\section{INTRODUCTION}

The binarity of stars is a topic of ongoing research, particularly in light of the plethora of exoplanets discovered over the past couple of decades. Exoplanets orbiting stars with a binary companion pose significant implications for formation theories, such as orbital stability (Holman \& Wiegert 1999), and the period-mass (Zucker \& Mazeh 2002) and periodeccentricity (Eggenberger et al. 2004) distributions. The searches for stellar companions to the host stars of Kepler exoplanet candidates have become an important component of the candidate validation process (Dressing et al. 2014; Everett et al. 2014; Wang et al. 2014). Attempts to detect binarity for the brightest exoplanet host stars are also underway (Crepp et al. 2012, 2013), and are often used to place constraints on additional planetary companions (Kane et al. 2014).

When it comes to multiplicity, one of the more exotic exoplanetary systems is that of 30 Arietus (hereafter 30 Ari). 30 Ari is a bound visual binary whose main components are both main sequence $\mathrm{F}$ stars (F5V and F6V) separated by 38". 1 $(1,500 \mathrm{AU})$. The $\mathrm{A}$ and $\mathrm{B}$ components are both relatively bright ( $V$ magnitudes of 6.48 and 7.09 respectively). 30 Ari A is a spectroscopic binary (Adams \& Joy 1919; Morbey \& Brosterhus 1974) with an orbital period of 1.1 days. 30 Ari B (HD 16232, HIP 12184, HR 764) was discovered by Guenther et al. (2009) to have a $\sim 10 M_{J}$ companion with an orbital period of 335 days. The discovery was made using radial velocity (RV) observations which are not easy to undertake for such an early-type star, despite its brightness. The reason for this is that the spectra of early-type stars have a relatively small number of absorption lines and also tend to have rapid rotation rates, thus inhibiting precision RV measurements. The brightness of 30 Ari B in close proximity to the equally bright $\mathrm{A}$ component also proves problematic for photometric observations and so the system remained relatively unobserved for the years following the exoplanet discovery. Recently 30 Ari B was revisited using the adaptive optics capabilities of the RoboAO system (Baranec et al. 2014) with target selection from the FG-67 database (Tokovinin 2014). The survey detected a stellar companion to the star (Riddle et al. 2015) that was further described by Roberts et al. (2015).

Here we present new observations of the 30 Ari B system that independently confirm the presence of a stellar companion in addition to the known planet orbiting the host star. Section 2 outlines the properties of 30 Ari B relevant to the subsequent analysis. Section 3 describes the detection of the stellar companion from Keck observations and the likelihood of the stars being bound. New RV and photometric data are presented in Section 4 which are both used to support the detection of the stellar companion and refine the properties of the known planet. Constraints on the physical and orbital properties of the stellar companion from these observations and orbital stability considerations are described in Section 5 . We provide concluding remarks in Section 6 including a discussion of names for the system components.

\section{HOST STAR PROPERTIES}

This paper compiles imaging, RV, and photometric data of the 30 Ari system. The advantage of combining these data sets is to maximize constraints on both the kinematic and intrinsic luminosities of the component members. Determining these properties of the individual components depends heavily on the properties of 30 Ari B. The fundamental stellar properties of the star have been published numerous times in the literature, most recently by Tsantaki et al. (2014). In order to compile a 
Table 1

30 Ari B Stellar Parameters ${ }^{\mathrm{a}}$

\begin{tabular}{|c|c|}
\hline Parameter & Value \\
\hline$J$ & 6.080 \\
\hline$V$ & 7.091 \\
\hline$B-V$ & 0.510 \\
\hline Proper motion $(\alpha, \delta)(\text { mas })^{\mathrm{b}}$ & $150.75,-12.79$ \\
\hline Parallax (mas) ${ }^{\mathrm{b}}$ & $24.52 \pm 0.68$ \\
\hline Distance $(\mathrm{pc})^{\mathrm{b}}$ & $40.8 \pm 1.1$ \\
\hline$M_{\star}\left(M_{\odot}\right)$ & $1.16 \pm 0.04$ \\
\hline$R_{\star}\left(R_{\odot}\right)$ & $1.13 \pm 0.03$ \\
\hline
\end{tabular}

Notes.

${ }^{a}$ Guenther et al. (2009) and references therein

b van Leeuwen (2007).

self-consistent set of stellar parameters relevant to this work, and for comparison with previous work on the planetary companion (see Section 4.1), we adopt those from van Leeuwen (2007) and Guenther et al. (2009), shown in Table 1. A particular reason for selecting these stellar parameters is to be consistent with the previous RV measurements of Guenther et al. (2009) such that a direct comparison of the Keplerian orbital solutions may be made (see Section 4).

\section{DETECTION OF A STELLAR COMPANION}

Shown in Figure 1 (left) is an $\sim 9^{\prime}$ FOV image of the 30 Ari visual binary extracted from the Digital Sky Survey ${ }^{11}$, centered on the A component. Our observations of 30 Ari B were acquired using NIRC2 with the AO system at Keck during the night of 2014 August 9th. We used the standard AO configuration for NIRC2 imaging observations, the details of which may be found in the NIRC2 Observer's Manual. ${ }^{12}$ Sky conditions were poor (thin cirrus clouds) but sufficient to complete the observations given the brightness of the target. The camera was used in the narrow camera mode with a $J$-band filter. A total of nine $0.2 \mathrm{~s}$ exposures were acquired and coadded to produce a combined smoothed frame from which to conduct the analysis. The sensitivity of the observations to fainter stellar companions is demonstrated in Figure 2 which shows the $5 \sigma$ detection limit as a function of radial separation from the host star.

The combined Keck image is shown in Figure 1 (right). 30 Ari $\mathrm{B}$ is at the center of the frame and the stellar companion is plainly visible to the right of the host star. Measurements of the stellar profile centroids and the NIRC2 pixel scale (0."009942/pixel) show that the stars are separated by $0 . " 536$. The uncertainty in the $X$-direction is 0.646 pixels, equivalent to 0 ". 00642 or 6.42 mas in R.A. Similarly the uncertainty in the $Y$-direction is 0.245 pixels, equivalent to 0 " 00244 or 2.44 mas in decl. Thus the separation of the stars is $0 . " 536 \pm 0$." 007 with a position angle of $-73^{\circ} .6 \pm 0.1$ (east of north).

The relative photometry between the two stars was estimated in two ways and the results compared. Because of the poor seeing on the night of the observations, there was not a clean centralized peak of the primary. To aid in the photometry, the final image was convolved with a $2 \mathrm{D}$ circularly symmetric Gaussian with the full-width set to 4 pixels: approximately half the full-width of the measured point-spread function (PSF). The

\footnotetext{
11 https://archive.stsci.edu/cgi-bin/dss_form

12 http://www2.keck.hawaii.edu/inst/nirc2/Manual/ObserversManual.html
}

first estimate utilized aperture photometry on each star where the aperture radius was set to the half-width of the convolved PSF (5 pixels). The second estimate was performed by fitting a 2D Gaussian to each of the stellar PSFs and subtracting the Gaussians from the image until the residuals were minimized. The total flux in the Gaussian PSFs were used to estimate the relative magnitudes of stars. The final relative photometry was determined from an average of the two methods, and the difference between the two methods was added in quadrature to the formal statistical uncertainties in the aperture and psf photometry. We find that the magnitude difference between the two stars $\Delta_{J}=3.15 \pm 0.07$. Using the distance estimate of Table 1 leads to a projected separation of $21.9 \pm 0.7 \mathrm{AU}$ and a companion absolute $J$ magnitude of $6.18 \pm 0.09$. This is consistent with the companion being a late-type dwarf with an approximate spectral type of M1-3 (Boyajian et al. 2012).

The issue remains as to whether the detected companion is indeed gravitationally bound to the host star. No astrometric motion was detected through the analysis of Hipparcos data by Reffert \& Quirrenbach (2011). This null-detection is not surprising however considering that the orbital period of the stellar companion is much longer than the time baseline of the Hipparcos observations. The proper motion of 30 Ari B according to van Leeuwen (2007) is 0 " $151 \pm 0$ ! 00075 . The astrometric results of Roberts et al. (2015) confirm that the newly detected companion to 30 Ari B has a common proper motion, increasing the likelihood that they are bound. To investigate this further, we adopt the statistical validation techniques described in Horch et al. (2014). The $5 \sigma$ detection limit shown in Figure 2 is similar to the detection limit achieved with the Differential Speckle Survey Instrument on Gemini-North, shown in Figure 9 of Horch et al. (2014). Linear interpolation of the figure bins indicates that the likelihood of our detected companion being bound to 30 Ari B is $>82 \%$. However, the observations of Horch et al. (2014) were of the Kepler field which has a higher density of stars. To account for that, we used the TRILEGAL code ${ }^{13}$ (Girardi et al. 2005) to determine the relative number of stars along the respective lines of sight for 30 Ari B and the Kepler field. A 1 square degree search with the TRILEGAL model yields 16,210 line of sight companions for 30 Ari B and 167,936 line of sight companions for the Kepler field $(l=76.53, b=13.29)$. Assuming the binarity rate does not change, the probability that the companion detected near 30 Ari B is gravitationally bound is increased by the ratio of the number of companions predicted, which is a factor of $\sim 10$. Thus, the probability that the detected companion is bound to 30 Ari B is $\sim 100 \%$.

At the point of submitting this work, we learned that the stellar companion to 30 Ari B has also been detected by the Robo-AO team (Riddle et al. 2015). We present the Keck AO component of these results as an independent detection of this companion. Their observations were conducted using an $i$ filter and reveal a similar angular separation of 0 ".536. If the detected companion is gravitationally bound, the 30 Ari B components should have colors and absolute magnitudes that are compatible with stellar isochrones. To test this, we combine the $J$-band detection from our Keck observations with the $i$-band detection from Robo-AO to place the components on a color-magnitude diagram.

\footnotetext{
13 http://stev.oapd.inaf.it/cgi-bin/trilegal
} 

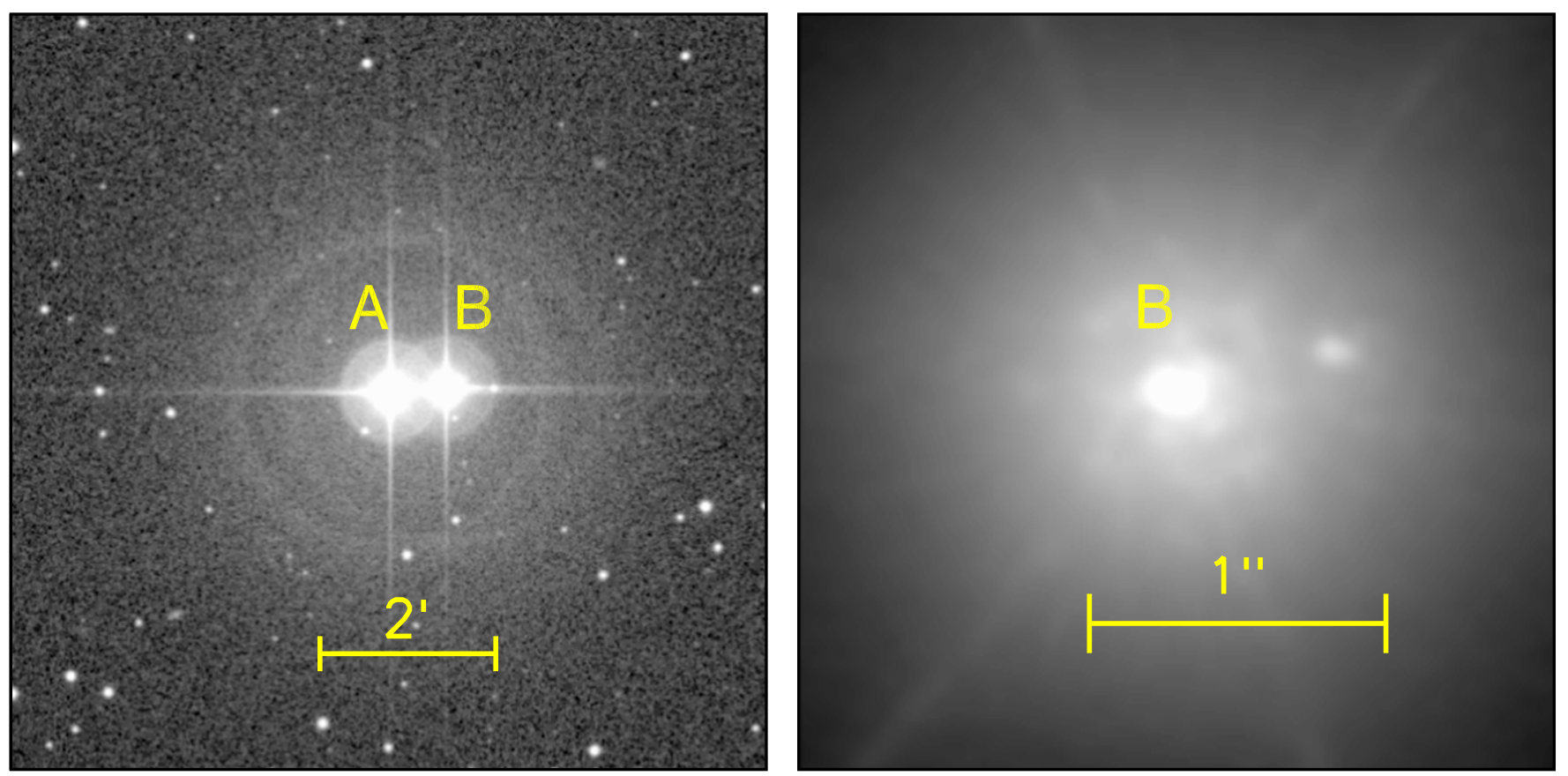

Figure 1. Images of the 30 Ari system from the Digital Sky Survey (left) and Keck observations (right). In both cases the field orientation is up-north and left-east. Left: image of the 30 Ari system centered on the A component. Right: Keck/NIRC2 combined image of 30 Ari B showing the presence of the stellar companion.

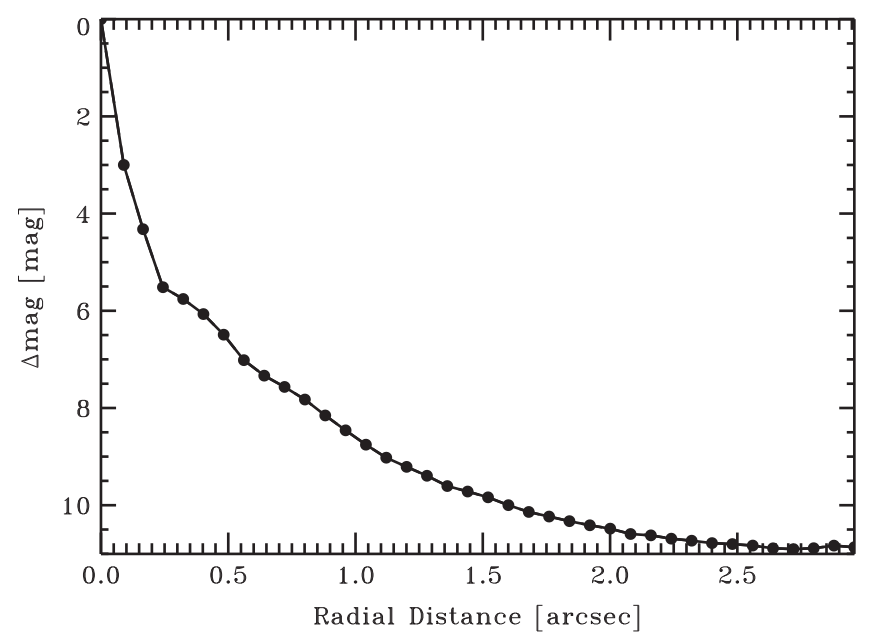

Figure 2. The $5 \sigma$ Keck image sensitivity (in units of $\Delta$ magnitude) as a function of separation from the host star.

The available $i$-band photometry of 30 Ari B from the Sloan (Ahn et al. 2012) and APASS $^{14}$ surveys is heavily saturated, and therefore not reliable for this system. To derive an approximate $i$ magnitude, we fit $J-K$ and $M_{J}$ to a grid of Dartmouth isochrones (Dotter et al. 2008) assuming zero reddening, which is justified by the relatively small distance ( $\sim 41 \mathrm{pc})$ to the system. The metallicity of the 30 Ari system is poorly constrained, with values ranging from near solar metallicity from Stromgren photometry (Casagrande et al. 2011) to $[\mathrm{Fe} / \mathrm{H}] \sim+0.27$ from various spectroscopic studies (see Guenther et al. 2009 and references therein). Adopting a metallicity prior of $[\mathrm{Fe} / \mathrm{H}]=0.1 \pm 0.2$ for the isochrone fit, which approximately corresponds to the central value and spread of the quoted literature values, we derive a

${ }^{14}$ http://www.aavso.org/apass

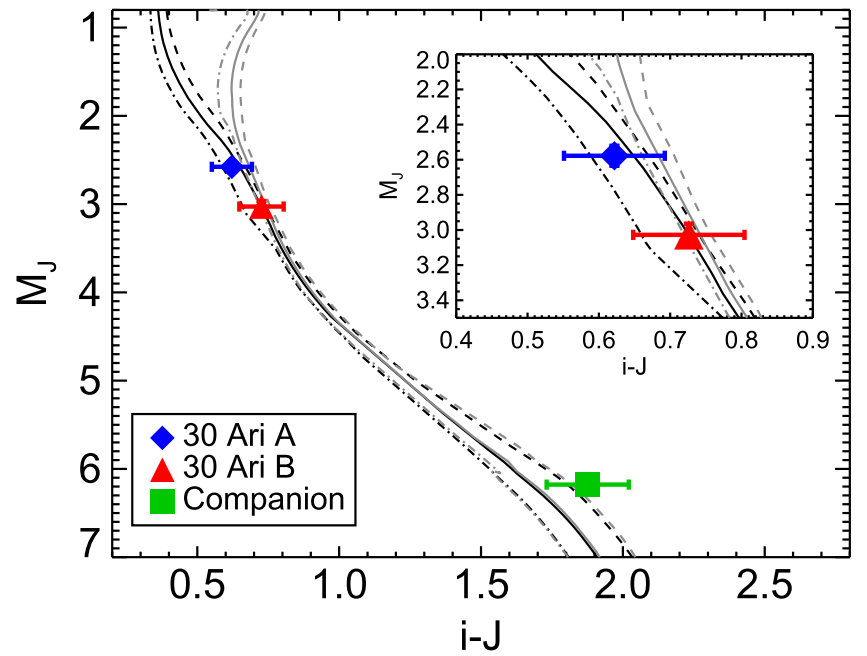

Figure 3. $0.5 \mathrm{Gyr}$ (black) and $1.5 \mathrm{Gyr}$ (gray) Dartmouth stellar isochrones with $[\mathrm{Fe} / \mathrm{H}]=-0.1$ (dashed-dotted lines), 0.1 (solid lines), and 0.3 (dashed lines). Note that the $0.5 \mathrm{Gyr}$ isochrone has been obtained by linearly interpolating the original $<1 \mathrm{Gyr}$ isochrone grid available in the Dartmouth database. The inset shows a zoom on the position of 30 Ari A and B.

synthetic absolute magnitude of $M_{i}=3.75 \pm 0.08$ mag for 30 Ari B. Combining this with $\Delta J=3.15 \pm 0.07 \mathrm{mag}$ and $\Delta$ $i=4.2 \pm 0.1 \mathrm{mag}$, the corresponding colors are $i-\mathrm{J}=$ $0.73 \pm 0.08 \mathrm{mag}$ for 30 Ari $\mathrm{B}$ and $i-\mathrm{J}=1.88 \pm 0.13 \mathrm{mag}$ for the detected stellar companion.

Figure 3 compares the positions of 30 Ari B and the detected companion in an $M_{J}, i-J$ color-magnitude diagram to 0.5-1.5 Gyr isochrones for a range of metallicities. 30 Ari A is also shown, with an $i-J$ color derived using the same procedure as described above. The comparison shows that all three components have colors that are consistent with a given distance modulus, and hence are compatible with being in a gravitationally bound system. 
Table 2

Keplerian Orbital Model

\begin{tabular}{lcc}
\hline \hline Parameter & $\begin{array}{c}\text { Value (Guenther } \\
\text { et al. 2009) }\end{array}$ & Value (This work) \\
\hline 30 Ari B b & $335.1 \pm 2.5$ & $345.4 \pm 3.8$ \\
$P$ (days) & $14538 \pm 20$ & $13222.1 \pm 42.4$ \\
$T_{\mathrm{p}(\mathrm{JD}-2,440,000)}$ & $0.289 \pm 0.092$ & $0.18 \pm 0.11$ \\
$e$ & $307 \pm 18$ & $337 \pm 57$ \\
$\omega(\mathrm{deg})$ & $272 \pm 24$ & $177 \pm 26$ \\
$K\left(\mathrm{~m} \mathrm{~s}^{-1}\right)$ & $9.88 \pm 0.94$ & $6.6 \pm 0.9$ \\
$M_{\mathrm{p}} \sin i\left(M_{J}\right)$ & $0.995 \pm 0.012$ & $1.01 \pm 0.01$ \\
$a(\mathrm{AU})$ & 0.0 & $-0.12 \pm 0.03$ \\
$d v / d t\left(\mathrm{~m} \mathrm{~s}^{-1}\right.$ day $\left.^{-1}\right)$ & $\ldots$ & \\
System Properties & $\ldots .8 \pm 17.7$ \\
$\gamma\left(\mathrm{m} \mathrm{s}^{-1}\right)$ & & \\
Measurements and $_{\quad}$ model $_{N_{\text {obs }}}$ & & 110 \\
rms $\left(\mathrm{m} \mathrm{s}^{-1}\right)$ & 98 & 181.5 \\
$\chi_{\text {red }}^{2}$ & 135 & 0.82 \\
\hline
\end{tabular}

\section{RADIAL VELOCITIES AND PHOTOMETRY}

Here we present new RV and photometric data of 30 Ari B in support of our observations of the stellar companion.

\subsection{Revised Planetary Parameters and Linear Trend}

The RV data set for 30 Ari B published by Guenther et al. (2009) consisted of 98 measurements and revealed the presence of a sub-stellar companion in a 335 day orbit around the host star. Guenther et al. (2009) did not provide a fit that included a linear trend free parameter since the presence of such a trend was negligible in those data. Here we provide 12 additional measurements which extend the time baseline by $\sim 300$ days and thus greater sensitivity to the possible influence of a stellar companion. The data were acquired from continued observations $2 \mathrm{~m}$ Alfred Jensch telescope of the Thüringer Landessternwarte Tautenburg, described in detail by Hatzes et al. (2005), and were reduced with the same data pipeline as for those for Guenther et al. (2009). The data were modeled using a partially linearized, least-squares fitting procedure (Wright \& Howard 2009). Parameter uncertainties were estimated using the BOOTTRAN bootstrapping routines developed by Wang et al. (2012). The new best-fit Keplerian orbital solution is shown in Table 2 and Figure 4 along with the fit residuals. The parameters include orbital period $(P)$, time of periastron passage $\left(T_{\mathrm{p}}\right)$, eccentricity $(e)$, periastron argument $(\omega), \mathrm{RV}$ semi-amplitude $(K)$, minimum planet mass $\left(M_{\mathrm{p}}\right)$, semimajor axis $(a)$, and the RV linear trend $(d v / d t)$. The complete set of 110 new and revised RV measurements is provided in Table 3.

There are several notable changes over the orbital solution of Guenther et al. (2009), shown in Table 2. The inclusion of a linear trend is warranted by the extended baseline and the solution shows that the trend is significant at the $4 \sigma$ level. The linear trend has consequences for the Keplerian solution in that the orbital period is slightly increased and the "shape" of the orbit (eccentricity and periastron argument) is less well constrained since it is closer to being circular. Another change of note is that the linear trend partially compensates for the semi-amplitude of the RV variations resulting in a smaller minimum mass for the sub-stellar companion of $6.6 M_{J}$. The companion in question is thus more likely to be planetary in nature than an object in the brown dwarf regime. Finally, it should be noted that we have not excluded any of the significant RV outliers (e.g., the measurement acquired at epoch 2,452,655.25, see Table 3). Testing such exclusions did not significantly impact the Keplerian orbital solution. The implications of the linear trend for the detected stellar companion to 30 Ari B are discussed in more detail in Section 5.1.

\subsection{Potential Planetary Transit}

With the detection of the low-mass stellar companion to 30 Ari $\mathrm{B}$, we undertook the task of acquiring photometry that may have indications of stellar variability. Variability studies of Kepler stars have shown that F-type stars tend to have much shorter variation periods, likely due to pulsations rather than the activity typical of low-mass stars (Ciardi et al. 2011; McQuillan et al. 2012).

The first photometric data source we examined was the photometry from the Hipparcos mission, shown in the top panel of Figure 5. These data demonstrate photometric stability at the $\sim 1 \%$ level. However, there are two significant outliers in the photometry indicating an $\sim 5 \%$ reduction in brightness of the host star. The most intriguing aspect of these two outliers is that they are separated by $\sim 693.8$ days - approximately twice the revised orbital period of the planet (see Section 4.1). If such variation were indeed due to the passage of the planet across the stellar disk, the depth appears to be too large. Additionally, the probability of the transit being detected in the sparsely sampled Hipparcos data is extremely low. Nevertheless, to investigate this further, we constructed a transit ephemeris based upon the Hipparcos photometry since these data yield greater timing precision than predictions based upon the RV data described in Section 4.1.

30 Ari B was observed using the $0.6 \mathrm{~m}$ telescope at the Peter van de Kamp Observatory, Swarthmore College on the nights of 2014 November 15th and 16th. Observations were conducted in good weather conditions using an $r^{\prime}$ filter and $10 \mathrm{~s}$ exposures. 30 Ari A provided a natural comparison star from which to perform relative photometry since it is similar in both brightness and color. Based upon the Hipparcos dips, a possible event was predicted for a JD of around 2,456,976. These data are shown in the middle and bottom panels of Figure 5. Though no event similar to that seen in the Hipparcos data was detected, the star was observed to be consistently stable at the level of a couple of millimags. These data also rule out significant stellar pulsations of periods less than $\sim 6 \mathrm{hr}$. It is certainly possible that the outlier measurements in the Hipparcos data set are simply spurious, but the curious coincidence with the orbital period of the planet leads us to encourage continued observations.

\section{ORBITAL DYNAMICS OF THE COMPANION}

The properties of the stellar companion may be further constrained from orbital dynamics considerations, as we describe in this section.

\subsection{Mass and Orbit}

The mass and separation of the stellar companion to 30 Ari B may be constrained from the linear trend detected in the RV data (see Section 4.1). The trend does not exhibit a "turn-around" point where the slope changes from negative to 

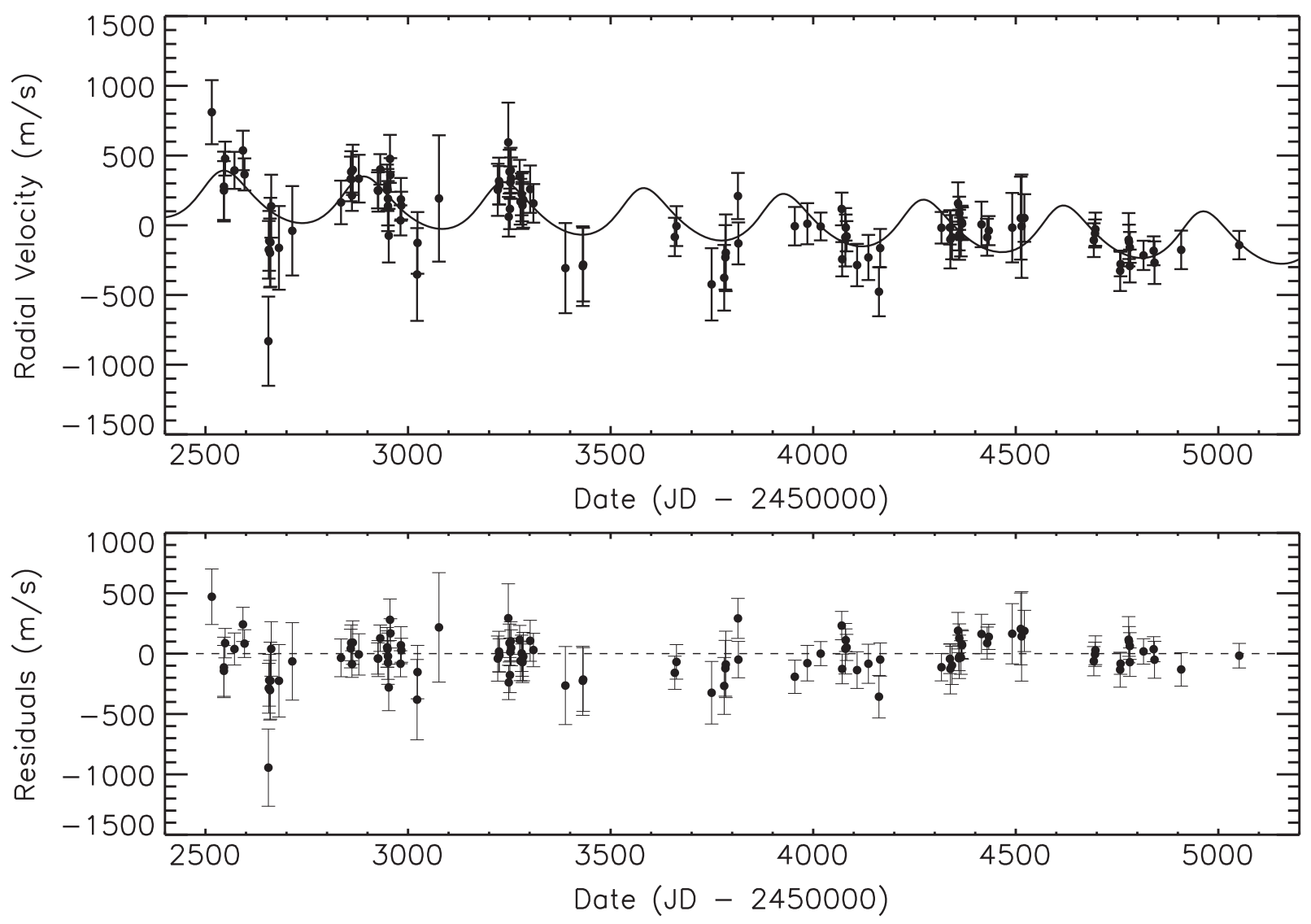

Figure 4. All $110 \mathrm{RV}$ measurements of 30 Ari B acquired using the $2 \mathrm{~m}$ Alfred Jensch telescope (see Table 3). Top: the new orbital solution including a linear trend due to the presence of the stellar companion, provided in Table 2. Bottom: the RV residuals (observed minus computed) from the best-fit model.

positive. However, the total amplitude of the trend over the time baseline of the observations places a lower limit on the semi-amplitude of the variations due to the companion. The trend shown in Table 2 multiplied by the time baseline $(2,536$ days) yields a minimum RV amplitude of $\sim 305 \mathrm{~m} \mathrm{~s}^{-1}$.

The left panel of Figure 6 shows the resulting mass/ separation limits where the linear trend has been converted to an acceleration, $\dot{v}$, and then converted to a mass estimate via $M_{B}=\left(\dot{v} a^{2}\right) / G$, where $\dot{v}=d v / d t, \quad M_{B}$ is the mass of the detected binary companion, and we have assumed a circular orbit. The limit is shown as a solid line and the dashed lines represent the $1 \sigma$ uncertainties propagated from the linear trend uncertainties. Anything below these lines has either insufficient mass or proximity to the host star to produce the observed trend. Since we know from the angular separation (see Section 3) that the companion semimajor axis is at least 21.9 AU (vertical dotted line), the companion lower mass limit is $\sim 27$ Jupiter masses. The valid area of parameter space shown in the figure may thus be constrained to the shaded region.

An additional constraint on the companion mass may be applied by extending the above methodology, as described by Howard et al. (2010). The physical separation between the 30 Ari B stars, if they are bound, is $21.9 \mathrm{AU} / \sin (\theta)$ where $\theta$ is the angle between our line of sight and the primary-secondary vector $(\theta=0$ implies the secondary is behind the primary). If the secondary is the source of the RV linear trend described in Section 4.1, then it imparts a line of sight acceleration of $\dot{v}=\left(G M_{B} / r^{2}\right) \times \cos (\theta)$ where $r$ is the physical separation with no assumption regarding orbital eccentricity. This leads to the following expression for the companion mass:

$$
M_{B}=\frac{\dot{v}(21.9 \mathrm{AU})^{2}}{G\left(1-\cos ^{2}(\theta)\right) \cos (\theta)} .
$$

The cubic term in the denominator must be negative since $d v /$ $d t$ is negative and $M_{B}$ is positive. Furthermore, because the cosine function is bound between -1 and 1 , the cubic's value lies between -0.385 and zero. The equation therefore becomes an inequality for $M_{B}$ :

$$
M_{B}>\frac{\left(-0.12 \pm 0.03 \mathrm{~m} \mathrm{~s}^{-1} \mathrm{day}^{-1}\right) \times(21.9 \pm 0.7 \mathrm{AU})^{2}}{G(-0.385)}
$$

where we have substituted the linear trend from Table 2. This results in a minimum mass of the stellar companion of $M_{b}>0.29 \pm 0.08 M_{\odot}$, consistent with the companion being M1-3 as determined in Section 3.

The calculated properties of the companion, including the mass of 30 Ari B and the projected separation, result in a minimum orbital period of $95 \pm 6$ years. This is consistent with the companion being of stellar mass producing an observed long timescale RV trend.

\subsection{System Orbital Stability}

The existence of a planet located $\sim 1$ AU from the host star may be used to place further constraints on the orbit of the stellar companion. Planets have been detected in both S-type 
Table 3

30 Ari B Radial Velocities

\begin{tabular}{|c|c|c|}
\hline $\begin{array}{l}\text { Date } \\
\text { (JD-2,450,000) }\end{array}$ & $\begin{array}{c}\mathrm{RV} \\
\left(\mathrm{m} \mathrm{s}^{-1}\right)\end{array}$ & $\begin{array}{c}\sigma \\
\left(\mathrm{m} \mathrm{s}^{-1}\right)\end{array}$ \\
\hline 2515.600993 & 754.1050 & 229.87 \\
\hline 2545.536478 & 220.9028 & 250.29 \\
\hline 2545.544754 & 192.3126 & 210.82 \\
\hline 2548.472892 & 421.2561 & 121.74 \\
\hline 2571.576275 & 336.9965 & 132.71 \\
\hline 2592.467015 & 479.7458 & 141.60 \\
\hline 2596.415295 & 307.9014 & 114.94 \\
\hline 2655.252792 & -888.2267 & 319.81 \\
\hline 2656.242768 & -232.6306 & 206.70 \\
\hline 2657.267185 & -168.8275 & 214.44 \\
\hline 2659.260606 & -255.4376 & 246.96 \\
\hline 2660.294048 & -178.7523 & 318.83 \\
\hline 2662.268544 & 79.8795 & 225.79 \\
\hline 2681.341841 & -219.0311 & 300.12 \\
\hline 2714.276746 & -96.3552 & 320.68 \\
\hline 2834.523535 & 107.0504 & 156.15 \\
\hline 2858.575149 & 275.5233 & 160.05 \\
\hline 2859.600468 & 326.0843 & 148.02 \\
\hline 2861.586390 & 156.6134 & 110.20 \\
\hline 2863.588342 & 340.7115 & 180.12 \\
\hline 2878.477610 & 277.3908 & 171.12 \\
\hline 2925.422421 & 193.6456 & 129.42 \\
\hline 2926.480403 & 190.0219 & 148.87 \\
\hline 2931.400890 & 343.9288 & 109.36 \\
\hline 2948.365052 & 213.9280 & 164.54 \\
\hline 2949.384915 & 196.2251 & 150.77 \\
\hline 2950.438364 & 134.0650 & 191.77 \\
\hline 2950.444684 & 80.5700 & 179.43 \\
\hline 2952.400062 & -131.2379 & 192.41 \\
\hline 2955.422149 & 419.4916 & 172.12 \\
\hline 2956.465067 & 303.5786 & 121.07 \\
\hline 2981.367219 & -21.7257 & 107.85 \\
\hline 2982.388335 & 129.1056 & 153.39 \\
\hline 2983.413199 & 84.5132 & 99.35 \\
\hline 3022.290291 & -409.6576 & 332.74 \\
\hline 3023.367630 & -183.1904 & 221.21 \\
\hline 3076.292073 & 135.4815 & 453.15 \\
\hline 3221.496697 & 197.4571 & 186.82 \\
\hline 3224.547386 & 260.7274 & 167.63 \\
\hline 3225.512468 & 229.8522 & 137.80 \\
\hline 3247.392319 & 537.8008 & 284.92 \\
\hline 3248.490970 & 4.2384 & 142.16 \\
\hline 3250.480289 & 327.6601 & 156.85 \\
\hline 3251.466370 & 60.7189 & 145.38 \\
\hline 3252.492894 & 250.7651 & 114.60 \\
\hline 3253.432338 & 337.5163 & 161.58 \\
\hline 3254.450110 & 280.9421 & 148.70 \\
\hline 3275.513744 & 292.7484 & 119.60 \\
\hline 3277.546322 & 112.2383 & 164.09 \\
\hline 3280.508460 & 165.4369 & 150.31 \\
\hline 3281.621507 & 105.3440 & 133.67 \\
\hline 3282.493267 & 85.4409 & 168.59 \\
\hline 3284.350643 & 126.1022 & 200.37 \\
\hline 3301.371752 & 202.2588 & 169.87 \\
\hline 3309.444008 & 100.2754 & 138.43 \\
\hline 3388.344614 & -364.0933 & 323.23 \\
\hline 3431.291948 & -349.9726 & 285.70 \\
\hline 3432.265935 & -338.8958 & 263.92 \\
\hline 3658.364855 & -140.6013 & 137.69 \\
\hline 3662.535227 & -62.5995 & 142.97 \\
\hline 3749.258017 & -480.4116 & 259.14 \\
\hline 3780.366708 & -433.3393 & 235.41 \\
\hline 3783.265338 & -286.6540 & 231.20 \\
\hline
\end{tabular}

Table 3

(Continued)

\begin{tabular}{|c|c|c|}
\hline $\begin{array}{l}\text { Date } \\
(\mathrm{JD}-2,450,000)\end{array}$ & $\begin{array}{c}\mathrm{RV} \\
\left(\mathrm{m} \mathrm{s}^{-1}\right)\end{array}$ & $\begin{array}{c}\sigma \\
\left(\mathrm{m} \mathrm{s}^{-1}\right)\end{array}$ \\
\hline 3784.288850 & -254.0487 & 275.03 \\
\hline 3814.276631 & 152.9526 & 165.55 \\
\hline 3815.290494 & -186.8709 & 150.55 \\
\hline 3954.589497 & -63.4947 & 137.82 \\
\hline 3985.625596 & -45.7626 & 147.38 \\
\hline 4018.454970 & -65.4861 & 99.70 \\
\hline 4070.394848 & 60.2101 & 117.30 \\
\hline 4071.515854 & -300.3802 & 122.88 \\
\hline 4079.374428 & -142.8664 & 209.14 \\
\hline 4080.365276 & -73.1703 & 93.38 \\
\hline 4082.438154 & -135.5155 & 108.22 \\
\hline 4108.340755 & -342.0903 & 151.94 \\
\hline 4136.254291 & -287.5546 & 161.89 \\
\hline 4162.258663 & -533.4807 & 176.15 \\
\hline 4165.348658 & -220.5430 & 137.41 \\
\hline 4316.580289 & -74.6582 & 113.25 \\
\hline 4337.564193 & -73.0027 & 121.86 \\
\hline 4338.559366 & -158.5779 & 207.82 \\
\hline 4342.615233 & -142.4315 & 158.33 \\
\hline 4357.560127 & 100.1291 & 151.16 \\
\hline 4359.572735 & -133.7737 & 103.00 \\
\hline 4360.541338 & -124.8448 & 179.69 \\
\hline 4360.576121 & 29.2391 & 118.99 \\
\hline 4364.560715 & -141.0313 & 139.21 \\
\hline 4366.536859 & -34.9721 & 118.68 \\
\hline 4367.554451 & -45.7453 & 117.28 \\
\hline 4415.427584 & -50.7634 & 163.37 \\
\hline 4429.384583 & -142.7605 & 132.56 \\
\hline 4433.367364 & -95.2091 & 104.31 \\
\hline 4491.292838 & -73.9298 & 249.26 \\
\hline 4512.358374 & -5.8152 & 297.29 \\
\hline 4514.353088 & -63.6416 & 370.58 \\
\hline 4521.285415 & -4.7234 & 170.85 \\
\hline 4692.598912 & -163.7491 & 120.98 \\
\hline 4695.601537 & -115.6291 & 100.32 \\
\hline 4696.539080 & -85.1723 & 119.14 \\
\hline 4757.636700 & -383.5377 & 144.69 \\
\hline 4758.610225 & -334.8741 & 87.91 \\
\hline 4778.588013 & -160.5203 & 191.33 \\
\hline 4779.531238 & -175.7052 & 123.25 \\
\hline 4781.531450 & -215.3962 & 110.56 \\
\hline 4781.573376 & -349.1836 & 118.00 \\
\hline 4815.330030 & -272.7974 & 104.98 \\
\hline 4840.347284 & -239.8999 & 103.61 \\
\hline 4842.460148 & -325.0461 & 152.85 \\
\hline 4908.311715 & -233.2704 & 138.19 \\
\hline 5051.539182 & -199.2144 & 101.76 \\
\hline
\end{tabular}

and P-type orbits, the stability of which have been investigated by numerous authors (Harrington 1977; Eggleton \& Kiseleva 1995; Musielak et al. 2005). We use the analytical solutions provided by Holman \& Wiegert (1999) to determine the range of binary separations and eccentricities that will allow the planetary orbit to remain stable. To do this, we invert Equation (1) of Holman \& Wiegert (1999) as follows

$$
\begin{aligned}
a_{b}= & a_{c} /[(0.464 \pm 0.006)]+(-0.380 \pm 0.010) \mu \\
& +(-0.631 \pm 0.034) e+(0.586 \pm 0.061) \mu e \\
& \left.+(0.150 \pm 0.041) e^{2}+(-0.198 \pm 0.074) \mu e^{2}\right]
\end{aligned}
$$



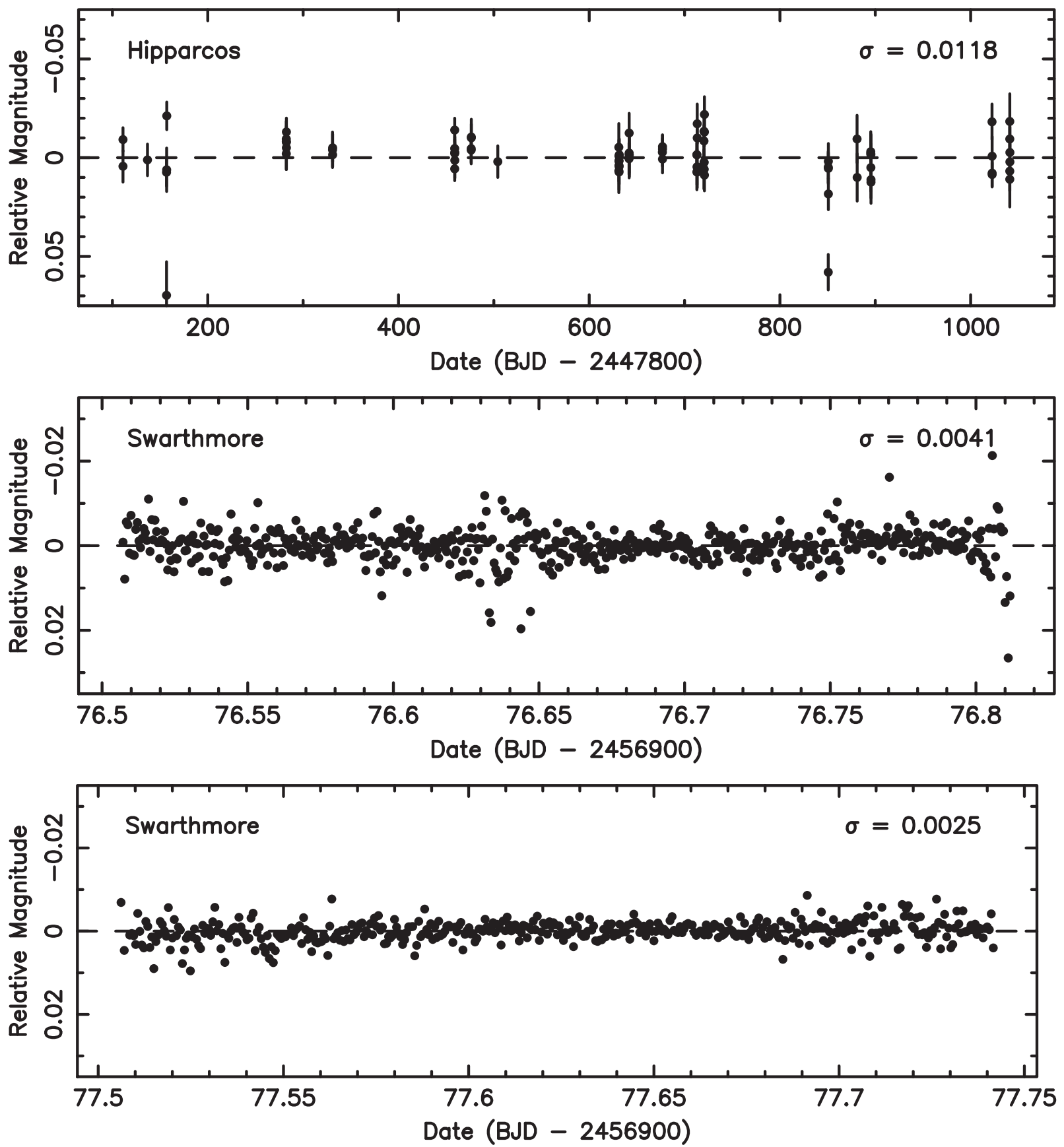

Figure 5. Photometry of 30 Ari B from the Hipparcos mission (top panel) and the Swarthmore $0.6 \mathrm{~m}$ telescope (middle and bottom panels). The number in the top right of each panel is the $1 \sigma \mathrm{rms}$ scatter of the data points. Though no transit events were observed in the Swarthmore data, the star was found to be photometrically stable within a couple of millimags. Note the different vertical axis scales between the Hipparcos and Swarthmore plots.

where $a_{\mathrm{b}}$ is the binary separation, $e$ is the binary orbital eccentricity, and $a_{c}$ is the maximum allowed semimajor axis of the planet. The mass ratio, $\mu$, is defined as $\mu=m_{2} /\left(m_{1}+m_{2}\right)$, and is thus $\mu=0.5$ for an equal mass binary. The right panel of Figure 6 shows the binary separation and eccentricity limitations for three different mass ratios with the constraint that a planet must be allowed to exist at $a_{c}=1.01 \mathrm{AU}$. We have the additional constraint imposed by the projected separation of 21.9 AU, represented by the horizontal dotted line. Based on our spectral type estimate of M1, we adopt a mass for the companion of $0.5 M_{\odot}$ resulting in a mass ratio of $\mu=0.3$. Thus, the valid regions of the plot exist above both the dotted and dashed lines. For a companion separation equal to the projected separation, the eccentricity of the binary orbit must be less than $\sim 0.75$.

\section{CONCLUSIONS}

In this paper, we have presented significant new observations that attempt to describe the detected objects orbiting 30 Ari B. Table 4 summarizes our derived parameters of the stellar companion to 30 Ari B. The 30 Ari system as a whole is clearly quite complex with the $\mathrm{A}$ and $\mathrm{B}$ components harboring planetary and stellar companions. This complexity may be attributed partially to the relative youth of the system since A 

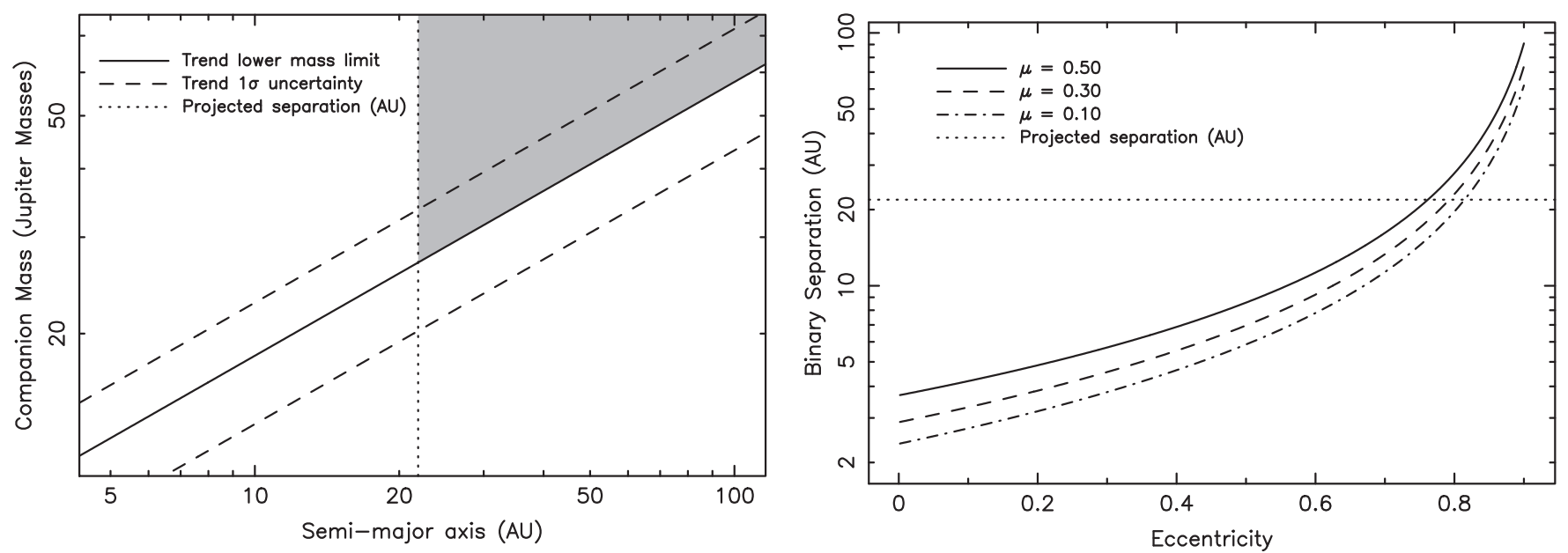

Figure 6. Limits on the properties of the companion star based on RV variations and stability constraints. Left: the lower mass limit for the stellar companion (solid line) imposed by the magnitude of the linear trend (assuming a circular orbit). The dashed lines are the $1 \sigma$ uncertainties and the vertical dotted line is the minimum projected separation (see Section 3). The shaded area is the valid region based on these constraints. Right: the minimum separation of the 30 Ari B binary components as a function of their orbital eccentricity that will allow the known planet to remain in a stable orbit. The horizontal dotted line represents the minimum projected separation of the stellar companion and the mass ratio is $\mu \sim 0.3$. Thus, the valid region of the plot is above both the dotted and dashed lines.

and B are each less than $1 \mathrm{Gyr}$ in age (Guenther et al. 2009), although the hierarchical structure of the system is likely stable for long timescales. Additionally, the relatively large (minimum) separation of the detected stellar companion to 30 Ari B produces orbital motion that makes it difficult to constrain the orbital inclination. If the companion and the known planet are coplanar then that would have significant implcations for the formation and evolutionary history of the system and provide additional constraints on the overall system stability. Further observations of the companion will be able to improve our knowledge of the inclination and the kinematics of the system.

The rather unusual nature of the system as described raises the issue of appropriate system component names. The reader will have noticed that we have thus far avoided assigning a name to the companion. The nomenclature of such systems is as complex as the system itself, an example of which is described by Wright et al. (2013). One possibility, which uses binary star and exoplanet naming conventions, would be to rename the primary and secondary components of 30 Ari B to 30 Ari $\mathrm{BA}$ and 30 Ari $\mathrm{BB}$, respectively, leading to a corrected name for the planetary companion of 30 Ari BA b. The guidelines of the Washington Multiplicity Catalog standard (Raghavan et al. 2010) recommend that the stellar components of 30 Ari B be named 30 Ari $\mathrm{Ba}$ and $30 \mathrm{Ari} \mathrm{Bb}$, leading to a collision with the planet naming convention. A compromise would be to name the newly detected companion 30 Ari $\mathrm{C}$ (also advocated by Roberts et al. 2015), allowing the planet to remain as $30 \mathrm{Ari} \mathrm{Bb}$. This would avoid having a name change for the planet, which is desirable from a literature paper-trail perspective. We propose to adopt the latter as a provisional naming convention for the system, as also adopted by Riddle et al. (2015). As it seems that many of the exoplanet host stars are part of binary systems, we can look forward to further discussion and adjustment of names and orbital parameters in the years ahead.

The authors would like to thank Elliott Horch, Steve Howell, and Suvrath Mahadevan for several useful discussions. Thanks
Table 4

Summary of Stellar Companion Properties

\begin{tabular}{lcc}
\hline \hline Parameter & Value & Section \\
\hline Angular separation $\left(^{\prime \prime}\right)$ & $0.536 \pm 0.007$ & 3 \\
Projected separation (AU) & $21.9 \pm 0.7$ & 3 \\
$\Delta J$ magnitude & $3.15 \pm 0.07$ & 3 \\
Apparent $J$ magnitude & $9.23 \pm 0.07$ & 3 \\
Absolute $J$ magnitude & $6.18 \pm 0.09$ & 3 \\
Spectral type & $\mathrm{M} 1-3$ & 3 \\
RV linear trend $\left(\mathrm{m} \mathrm{s}^{-1} \mathrm{day}^{-1}\right)$ & $-0.12 \pm 0.03$ & 4.1 \\
Mass $\left(M_{\odot}\right)$ & $>0.29 \pm 0.08$ & 5.1 \\
Orbital period (years) & $<95 \pm 6$ & 5.1 \\
Orbital eccentricity $\left(a_{b}=21.9 \mathrm{AU}\right)$ & $<0.75$ & 5.2 \\
\hline
\end{tabular}

are also due to the anonymous referee whose helpful comments improved the manuscript. D.H. acknowledges support by the Australian Research Council's Discovery Projects funding scheme (project number DE140101364) and by NASA under Grant NNX14AB92G issued through the Kepler Participating Scientist Program. E.V.Q. is supported by a NASA Senior Fellowship at the Ames Research Center, administered by Oak Ridge Associated Universities through a contract with NASA. This work made use of the Digitized Sky Survey (DSS) hosted by the Mikulski Archive for Space Telescopes (MAST). This work was supported by a NASA Keck PI Data Award, administered by the NASA Exoplanet Science Institute. Data presented herein were obtained at the W. M. Keck Observatory from telescope time allocated to the National Aeronautics and Space Administration through the agencys scientific partnership with the California Institute of Technology and the University of California. The Observatory was made possible by the generous financial support of the W. M. Keck Foundation. The authors wish to recognize and acknowledge the very significant cultural role and reverence that the summit of Mauna Kea has always had within the indigenous Hawaiian community. We are most fortunate to have the opportunity to conduct observations from this mountain. 


\section{REFERENCES}

Adams, W. S., \& Joy, A. H. 1919, ApJ, 49, 186

Ahn, C. P., Alexandroff, R., Allende Prieto, C., et al. 2012, ApJS, 203, 21

Baranec, C., Riddle, R., Law, N. M., et al. 2014, ApJL, 790, L8

Boyajian, T. S., von Braun, K., van Belle, G., et al. 2012, ApJ, 757, 112

Casagrande, L., Schönrich, R., Asplund, M., et al. 2011, A\&A, 530, 138

Ciardi, D. R., von Braun, K., Bryden, G., et al. 2011, AJ, 141, 108

Crepp, J. R., Johnson, J. A., Howard, A. W., et al. 2012, ApJ, 761, 39

Crepp, J. R., Johnson, J. A., Howard, A. W., et al. 2013, ApJ, 774, 1

Dotter, A., Chaboyer, B., Jevremović, D., et al. 2008, ApJS, 178, 89

Dressing, C. D., Adams, E. R., Dupree, A. K., Kulesa, C., \& McCarthy, D. 2014, AJ, 148, 78

Eggenberger, A., Udry, S., \& Mayor, M. 2004, A\&A, 417, 353

Eggleton, P., \& Kiseleva, L. 1995, ApJ, 455, 640

Everett, M. E., Barclay, T., Ciardi, D. R., et al. 2015, AJ, 149, 55

Girardi, L., Groenewegen, M. A. T., Hatziminaoglou, E., \& da Costa, L. 2005, A\&A, 436, 895

Guenther, E. W., Hartmann, M., Esposito, M., et al. 2009, A\&A, 507, 1659

Harrington, R. S. 1977, AJ, 82, 753

Hatzes, A. P., Guenther, E. W., Endl, M., et al. 2005, A\&A, 437, 743
Holman, M. J., \& Wiegert, P. A. 1999, AJ, 117, 621

Horch, E. P., Howell, S. B., Everett, M. E., \& Ciardi, D. R. 2014, ApJ, 795, 60

Howard, A. W., Johnson, J. A., Marcy, G. W., et al. 2010, ApJ, 721, 1467

Kane, S. R., Howell, S. B., Horch, E. P., et al. 2014, ApJ, 785, 93

McQuillan, A., Aigrain, S., \& Roberts, S. 2012, A\&A, 539, 137

Morbey, C. L., \& Brosterhus, E. B. 1974, PASP, 86, 455

Musielak, Z. E., Cuntz, M., Marshall, E. A., \& Stuit, T. D. 2005, A\&A, 434,355

Raghavan, D., McAlister, H. A., Henry, T. J., et al. 2010, ApJS, 190, 1

Reffert, S., \& Quirrenbach, A. 2011, A\&A, 527, A140

Riddle, R. L., Tokovinin, A., Mason, B. D., et al. 2015, ApJ, 799, 4

Roberts, L. C., Tokovinin, A., Mason, B. D., et al. 2015, AJ, 149, 118

Tokovinin, A. 2014, AJ, 147, 86

Tsantaki, M., Sousa, S. G., Santos, N. C., et al. 2014, A\&A, 570, 80

van Leeuwen, F. 2007, A\&A, 474, 653

Wang, J., Fischer, D. A., Xie, J.-W., \& Ciardi, D. R. 2014, ApJ, 791, 111

Wang, S. X., Wright, J. T., Cochran, W., et al. 2012, ApJ, 761, 46

Wright, J. T., \& Howard, A. W. 2009, ApJS, 182, 205

Wright, J. T., Roy, A., Mahadevan, S., et al. 2013, ApJ, 770, 119

Zucker, S., \& Mazeh, T. 2002, ApJL, 568, L113 